\title{
Development of a real-time motor-imagery-based EEG brain-machine interface
}

\author{
Gal Gorjup ${ }^{1}$, Rok Vrabič ${ }^{1}$, Stoyan Petrov Stoyanov ${ }^{2}$, Morten Østergaard \\ Andersen $^{3}$, and Poramate Manoonpong ${ }^{4,5}$ \\ 1 Faculty of Mechanical Engineering, University of Ljubljana, Slovenia \\ gorjup.gal@gmail.com, rok.vrabic@fs.uni-lj.si \\ 2 Berger Neurorobotics, Odense, Denmark \\ stoyan@bergerneurorobotics.com \\ ${ }^{3}$ SDU Biotechnology, The University of Southern Denmark, Odense, Denmark \\ moan@kbm.sdu. dk \\ 4 The Maersk Mc-Kinney Møller Institute, The University of Southern Denmark, \\ Odense, Denmark \\ poma@mmmi.sdu.dk \\ ${ }^{5}$ IST, Vidyasirimedhi Institute of Science and Technology, Rayong, Thailand
}

\begin{abstract}
EEG-based brain-machine interfaces offer an alternative means of interact with the environment relying solely on interpreting brain activity. They can not only significantly improve the life quality of people with neuromuscular disabilities, but also present a wide range of opportunities for industrial and commercial applications. This work focuses on the development of a real-time brain-machine interface based on processing and classification of motor imagery EEG signals. The goal was to develop a fast and reliable system that can function in everyday noisy environments. To achieve this, various filtering, feature extraction, and classification methods were tested on three data sets, two of which were recorded in a noisy public setting. Results suggested that the tested linear classifier, paired with band power features, offers higher robustness and similar prediction accuracy, compared to a non-linear classifier based on recurrent neural networks. The final configuration was also successfully tested on a real-time system.
\end{abstract}

Keywords: electroencephalography, brain-machine interface, brain-computer interface, motor imagery, digital filtering, feature extraction, classification

\section{Introduction}

Electroencephalography (EEG) is a non-invasive method for recording electrical activity of the brain, where the data is collected by means of electrodes positioned on the scalp. The obtained voltage signal is very weak and requires amplification before digital conversion and storage. Further steps generally consist of noise reduction procedures, followed by methods for interpreting the obtained data which are governed by the target application (Fig. 1). 
In the case of EEG based brain-machine interfaces (BMI), the goal is to decode the user's intent from the recorded signals. This allows the user to interact with his/her environment without using the default neuromuscular pathways. Such interfaces are mainly used to help people whose medical conditions hinder their muscle manipulation abilities, but alternative uses (e.g. mental state monitoring) are also being actively developed.

The structure of a generic EEG-based brain-machine interface can be seen in Fig. 1. The brain signals are captured and digitised through EEG recording equipment. The general approach for the signal processing is to first filter out the noise and artefacts, then extract relevant features, and finally feed them to a trained classifier that produces a prediction of the user's intent. This classification output can then be used to control an arbitrary system that may also send feedback to the user. Several EEG-based brain-machine interfaces have already been developed [23,12], exploiting different control mechanisms.

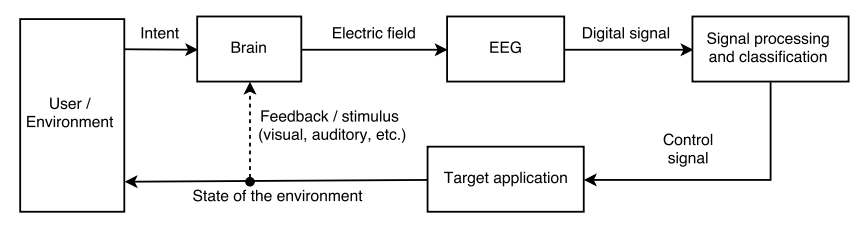

Fig. 1. Structure of a generic EEG-based brain-machine interface.

An EEG based BMI can be based on different signal features that can be manipulated and controlled by the user through a set of mental strategies. This work focuses on frequency features of the signals (brain rhythms), which are associated with activity levels within different areas of the brain cortex [4]. The rhythms can be detected in localised frequency bands over different regions of the scalp. The BMI developed in this work uses rhythms detected over the motor cortex. The rhythms exhibit changes in amplitude related to real or imagined movement of various body parts $[14,17]$. Through the mental strategy of motor imagery (MI), these amplitude changes can be voluntarily triggered by imagining limb movement, most commonly of the hands and feet. Several interfaces based on this principle have already been developed [16, 22, 5].

However, most motor imagery based brain-machine interfaces rely on laboratory conditions and professional recording equipment with wet electrodes to produce results with high reliability and prediction accuracy $[16,20,18]$. This work attempted to place the interface in a real world setting by testing a wide array of signal processing and classification methods not only on data sets recorded in laboratory conditions, but also on sets that contain notable amounts of noise. In addition, it explored the usage of novel classifiers based on recurrent neural networks that can process sequential temporal features. The best performing methods were in the end also tested in a noisy environment using compact, low 
cost hardware with dry electrodes where the goal was to determine a possibility of using the simple and reliable real-time interface for everyday use.

This article first presents the used data sets and software framework in sections 2 and 3, respectively. Sections 4 and 5 introduce the implemented filtering, feature extraction and classification methods used for processing the motor imagery EEG signals. The implemented methods are then tested and evaluated in section 6 with respect to the research goals. Section 6.3 presents real-time operation of the system with dry electrodes using previously found optimal processing methods and section 7 provides conclusion of this work.

\section{Data Set Descriptions}

For offline testing and method selection, three motor imagery data sets were used.

The first used data set was recorded for the purposes of a BCI competition [6], and was publicly available online. It contains left hand, right hand, legs and tongue MI trials and was recorded in a monopolar fashion. $288 \mathrm{MI}$ trials were recorded per person (72 per MI class) and only two of the 9 subjects were used in this work to match the size of the following data sets. The selected subjects correspond to the two presented in more detail in [13].

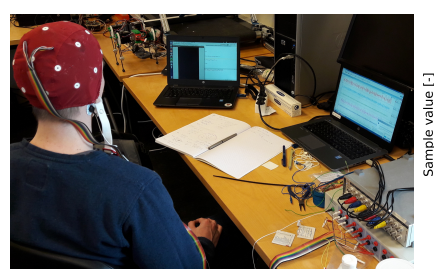

(a)

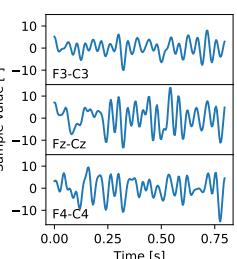

Time [s]

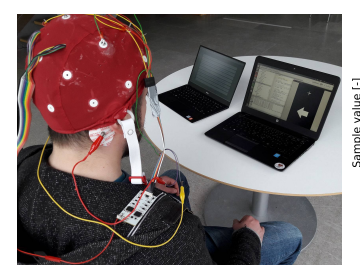

(b)

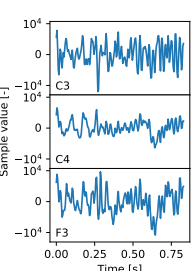

Fig. 2. Recording setup and sample recorded signals for (a) bipolar wet data and (b) monopolar dry data.

The second set was recorded for the purposes of this work and contains only left and right hand MI classes. It was recorded in a bipolar fashion, using wet electrode pairs F7-T3, F3-C3, Fz-Cz, F4-C4, and F8-T4, with respect to the international 10-20 system [11] (Fig. 2a). The signals were captured using an MLAEC1 EEG Electro-Cap System, an ML138 Octal Bioamp and a PowerLab 16/35 data acquisition system (all from AD Instruments). The set consists of two subjects and 96 trials per subject (48 per MI class).

The third set was also recorded within the frame of this work and again contains left and right hand MI classes. It was recorded in a monopolar fashion using dry electrodes at locations C3, C4, F3 and F4 (according to the international 10-20 system) with ear reference (Fig. 2b). The set consists of 192 trials 
per subject, 96 per MI group, recorded on two subjects. Hardware details are listed in section 6.3.

The second and third data set were recorded in the entrance hall to an engineering research building - an environment containing electromagnetic noise, as well as potential distractions for the subjects in the form of random by-passers and their conversations.

In all of the recordings, the MI trials follow the timing scheme presented in Fig. 3. Each trial started with a fixation cross appearing on the screen, which prompted the subject to focus. After $2 \mathrm{~s}$, a cue indicating which class of motor imagery the subject should perform appeared on screen. The subjects were asked to perform motor imagery until the fixation cross disappeared at $t=6 \mathrm{~s}$. After a short break, the next trial initiated. No feedback was provided to the subjects while recording.

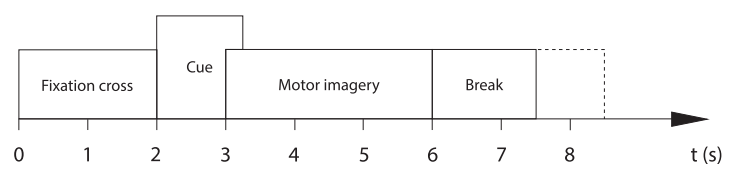

Fig. 3. Reference data trial timing scheme. Image adapted from [6].

\section{Software Framework}

In this work, a software framework was developed to support implementations of different processing methods. It was developed as a set of class hierarchies in the Python language. It was chosen because it is user friendly and well supported in terms of libraries that provide high level data structures and tools for signal processing and machine learning. Speed was not a primary objective as the brain rhythm changes are relatively slow and highly optimised processing algorithms would not significantly improve the overall response time.

\section{Signal Processing}

A traditional approach to EEG signal processing was taken, where filtering, feature extraction, and classification methods were implemented and evaluated. The methods were developed to support multivariate temporal data as all of the data sets contained recordings from multiple channels.

\subsection{Signal Filtering}

The family of digital temporal filters was considered for the purpose of reducing the amount of noise in the signals, as well as for isolating the frequency bands of interest from the recordings. 
Even though finite impulse response (FIR) filters have been suggested for scientific EEG signal processing purposes [21], infinite impulse response (IIR) filters were found to be more appropriate in this setting. They offer significantly lower delay times than FIR filters, which greatly influences the responsiveness of the system. Although FIR filters are always stable, IIR instability issues can be easily avoided with proper design $[21,3]$. The filtered signals are not recorded for the purpose of detailed inspection but are instead passed to various machine learning techniques. Minor signal distortions therefore do not significantly affect the results, as long as the same filter is applied to all of the processed data.

\subsection{Feature Extraction Methods}

Features can be extracted from the time- or frequency-domain of the EEG signal. The choice of extraction methods depends on where the sought information is encoded. In this case, the implemented features were focused on the frequency domain. Four methods were investigated: Band power, Autoregressive, Hjorth parameters, and FFT features.

1) Band power features rely on isolating a frequency band of interest from the signal using a band-pass filter and then squaring and averaging the result to obtain a temporal power signal in that band. These features were already extensively used in EEG experiments [14,15], giving solid results. They are computationally inexpensive and offer a very high temporal resolution which is equal to the signal's sampling rate. In this case, the features were constructed using a set of 5th order band-pass Butterworth IIR filters in frequency bands $8-12 \mathrm{~Hz}$, $12-16 \mathrm{~Hz}$ and $16-28 \mathrm{~Hz}$, where the first and last band correspond to mu and beta brain rhythm frequencies that hold information about imagined movement.

2) Autoregressive (AR) modelling is a parametric spectral method, meaning that it encodes spectral information into a selected number of parameters [3]. Those parameters are therefore very appropriate for distinguishing between signals that differ in the frequency spectrum, such as motor imagery recordings. The method implementation computes estimates of the AR coefficients by solving the Yule-Walker equations directly [3], using biased estimates for the autocorrelation sequence. The optimal number of AR parameters per EEG channel was here found to be 7 , according to the Akaike Information Theoretic Criterion [3] and result inspection.

3) Hjorth parameters [9] can easily be calculated in the time domain, but also contain information from the frequency domain, making them suitable for this application. For each of the EEG channels, three parameters were computed.

4) The Fast Fourier Transform (FFT) was the basis for the last set of features. Segments of separate EEG channels were transformed and then averaged in frequency bands of interest or output as a whole sequence.

\section{Classification Methods}

Two classification methods were implemented in the work and their training was window based: the MI trials (refer to Fig. 3) were split into windows of length 
$1 \mathrm{~s}$, with an $80 \%$ overlap. The windows whose centres fell between 4.5 and $5.5 \mathrm{~s}$ in the trial scheme were isolated and used for classifier training, after extracting the relevant features. Trial splitting, as well as feature extraction and training was performed simultaneously on all the relevant channels.

\subsection{Linear classifier}

The first classification method was based on the linear discriminant analysis (LDA) classifier [8] and also served as a benchmark for system validation, comparing it to results obtained in [13]. The one-versus-rest classification scheme was used, where a separate LDA classifier was trained for each of the MI groups.

\subsection{Neural network based nonlinear classifier}

The second method was based on recurrent neural networks, chosen by their ability to process sequences and produce non-linear decision boundaries. The core of the networks was Long Short-Term Memory (LSTM) units, which were designed to solve the vanishing gradient problems in sequence modelling through their gated structure. The architecture of an example LSTM based classifier can be seen in Fig. 4. The figure presents an unfolded architecture, showing how the network processes the sequences through time. On the lowest level, vectors from the input $\boldsymbol{x}$ are passed to the first LSTM layer $\boldsymbol{L}_{1}$, which passes its current results to the second LSTM layer $\boldsymbol{L}_{2}$ (each circle in the figure represents an array of units). In the next time step, the first layer receives a new vector from the input sequence and its own recurrent connection (same holds for the second layer). When the whole sequence has passed through the $\boldsymbol{L}_{1}$ and $\boldsymbol{L}_{2}$ layers, a fully connected layer $\boldsymbol{f}$ gathers the results from separate LSTM units in $\boldsymbol{L}_{2}$ into the appropriate number of MI classes $y$. The network was trained through

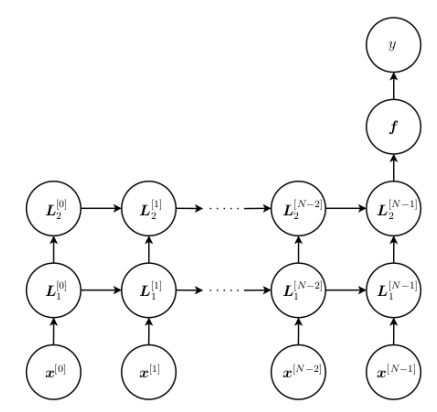

Fig. 4. Unfolded neural classifier with two LSTM layers.

gradient-based optimisation of the chosen categorical cross-entropy loss function [7]. The optimisation algorithm used was Adam [10], which is an adaptive learning rate algorithm with incorporated momentum. The network was trained in 
minibatches of 50 trials for 200 epochs. To prevent overfitting, the weight decay regularisation strategy [7] was applied to weights in the input and recurrent connections of the LSTM layers, as well as on the final fully connected layer.

In the design, several network hyperparameters were left to be optimised: number of LSTM layers, number of units in each layer, initial learning rate for the Adam algorithm and three weight decay parameters. These parameters were found through a massive random search approach that required extensive parallelisation over roughly $500 \mathrm{CPU}$ units in a cluster computing network (SLING [2]). The search was executed in two stages, where the first found coarse parameter values and the second refined them. For every data set presented in section 2 and every feature group described in section 4, roughly 2500 networks with random hyperparameter values (in defined limits) were trained and cross-validated (5-fold in the first stage, 10-fold in the second) to find relevant prediction accuracy. The prediction accuracy was used as a basis for selecting the optimal hyperparameter values. The overall network performances were compared and optimal hyperparameter values were found to be: 1 LSTM layer with 50 units, Adam learning rate in interval $\left[10^{-3.9}, 10^{-3.3}\right]$, LSTM input and recurrent connection weight decay in interval $\left[10^{-5}, 10^{-2}\right]$ and the final fully connected layer weight decay of $10^{-3.5}$.

\section{Experimental Results}

\subsection{Feature extractor selection}

In the first stage, all of the implemented feature extraction methods were tested with both classifiers. For the neural classifier, the tests were implicitly performed during the hyperparameter optimisation process and it was found that the feature group of band power sequences in ranges of $8-12 \mathrm{~Hz}, 12-16 \mathrm{~Hz}$, and 16-28 $\mathrm{Hz}$ gave the best performance.

For the linear classifier, feature test results for subjects 1 and 2 from the publicly available data set can be seen in Fig. 5 . The figure presents the 5 tested feature groups (band power, autoregressive, Hjorth parameters, FFT features and their combination), with respect to their average prediction accuracy and standard deviation obtained through 10 -fold cross validation with the linear classifier. In this case, the band power features (ranges of 8-12 Hz, 12-16 Hz and $16-28 \mathrm{~Hz}$ ) gave highest prediction accuracy with small variance.

The results showed that the band power features offer the best prediction accuracy over all data sets and both classifiers. Considering also their robustness and low computational complexity, they were chosen as the default feature extraction method for a real-time motor-imagery-based EEG brain-machine interface.

\subsection{Classifier selection}

In the second stage, the implemented classifiers, paired with the selected band power features from the previous section, were compared with the goal of determining which is the most appropriate for the available types of data and 


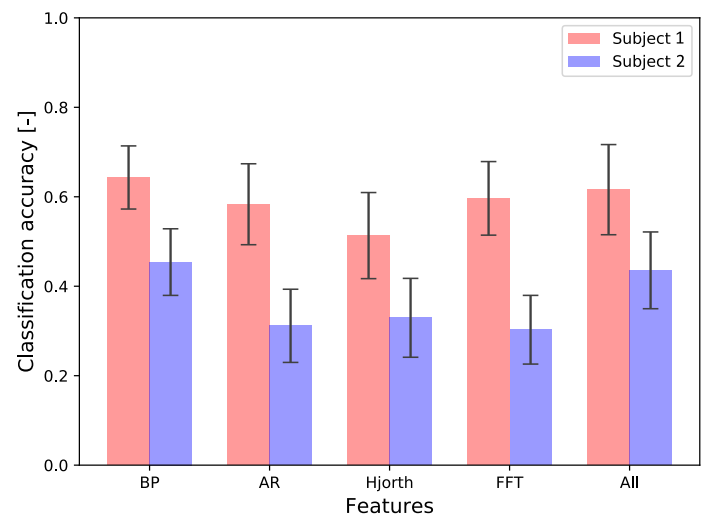

Fig. 5. Linear classifier performance with respect to different feature extraction methods for the publicly available data set. The methods include band power (BP), autoregressive (AR), Hjorth parameters (Hjorth), FFT features (FFT) and their combination (All).

training paradigm. The classifiers were trained and tested on the 6 subjects from the three available data sets described in section 2 . The MI trials were split into windows of length $1 \mathrm{~s}$, with an $80 \%$ overlap. Windows with centres between 4.5 and $5.5 \mathrm{~s}$ in the trial scheme were used for training the classifiers. Unbiased temporal prediction accuracies were obtained through 10 -fold cross validation. Example comparisons for subjects from different data sets can be seen in Fig. 6. The plots represent averaged classification accuracy through the trials (refer to trial scheme in Fig. 3) and the shaded blue area indicates its standard deviation. The vertical red and cyan lines indicate the cues that prompted the user to start and stop imagined movement. Before the MI start, classification accuracy moves around $25 \%$ and $50 \%$ for the subjects. These two percentages correspond to random guessing between 4 and 2 MI classes, respectively.

Inspecting results presented in Fig. 6, as well as those from the other subjects, it was found that the linear classifier is a more appropriate choice for the interface. It offers the same or better performance than the neural classifier at significantly lower complexity. It is also robust, deterministic and allows faster training through its closed-form optimisation solution.

The neural classifier, on the other hand, has a longer training time due to its nonlinear nature and the chosen iterative optimisation methods. The classifier's performance also substantially depends on hyperparameter values which take quite some time and effort to determine for a specific problem. However, the neural classifier may have the potential to outperform the linear if a different training approach was taken and more data was available. 

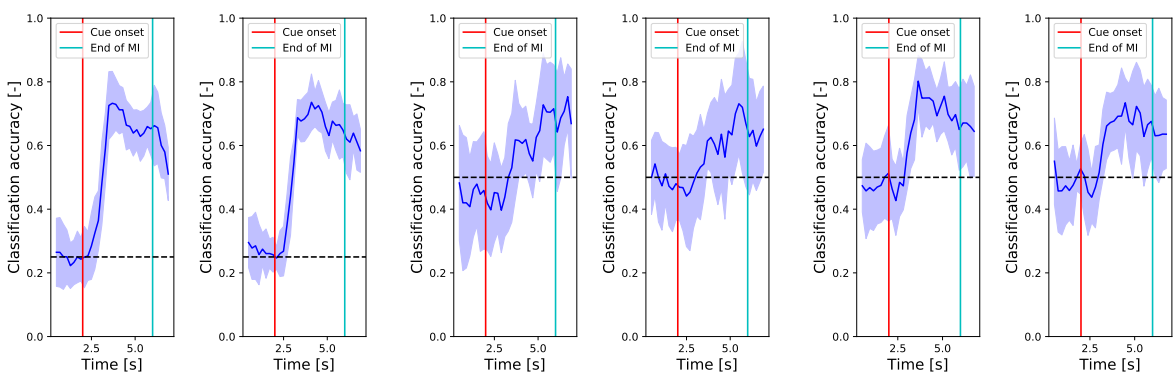

(a) Linear (left) and neu- (b) Linear (left) and neu- (c) Linear (left) and neural (right) classifier temporal ral (right) classifier temporal ral (right) classifier tempoaccuracy plots using the BP accuracy plots using the BP ral accuracy plots using the feature for subject 1 from feature for subject 1 from BP feature for subject 2 the publicly available data the recorded bipolar data set from the recorded monoposet. with wet electrodes. lar data set with dry electrodes.

Fig. 6. Comparison of linear and neural classifier performance.

\subsection{Real-time motor imagery based control}

The experiment was performed by one untrained, able-bodied male subject aged 24 , who has previously participated as subject 2 in the last recorded data set. The test was based on two class motor imagery, where the subject imagined movement of his left and right hands. The EEG signal was captured with dry reusable electrodes from Florida Research Instruments, mounted at locations C3 and C4 (according to the international 10-20 system) in monopolar montage with reference on the left and ground on the right mastoid. The signals were amplified and digitised with a battery powered prototype board developed by Berger Neurorobotics [1], which was streaming the data to a dedicated computer through a Bluetooth connection. The board had a fixed amplifier sensitivity of $100 \mu \mathrm{V}$ and a sampling frequency of $240 \mathrm{~Hz}$. The setup can be seen in Fig. 2b.

Taking into account the findings in Section 6, the linear one-versus-rest LDA classifier was chosen to be used in the tests, along with the band power features in ranges of $8-12 \mathrm{~Hz}, 12-16 \mathrm{~Hz}$ and $16-28 \mathrm{~Hz}$. The classifier was trained on data from subject 2 in the dry data set, using the training paradigm described in Section 6.2 and channels C3 and C4.

The trained classifier, along with required supporting signal processing methods and framework functionality, was then ported to the dedicated computer receiving the live data stream. The received data was accumulated and passed to the classifier in segments of length $1 \mathrm{~s}$ with an $80 \%$ overlap, mirroring its window-based training paradigm. With this setting, the classifier could produce a new label every $0.2 \mathrm{~s}$. To increase system stability, the classifier was expanded with functionality to refrain from predicting a class label if its confidence was too low. 

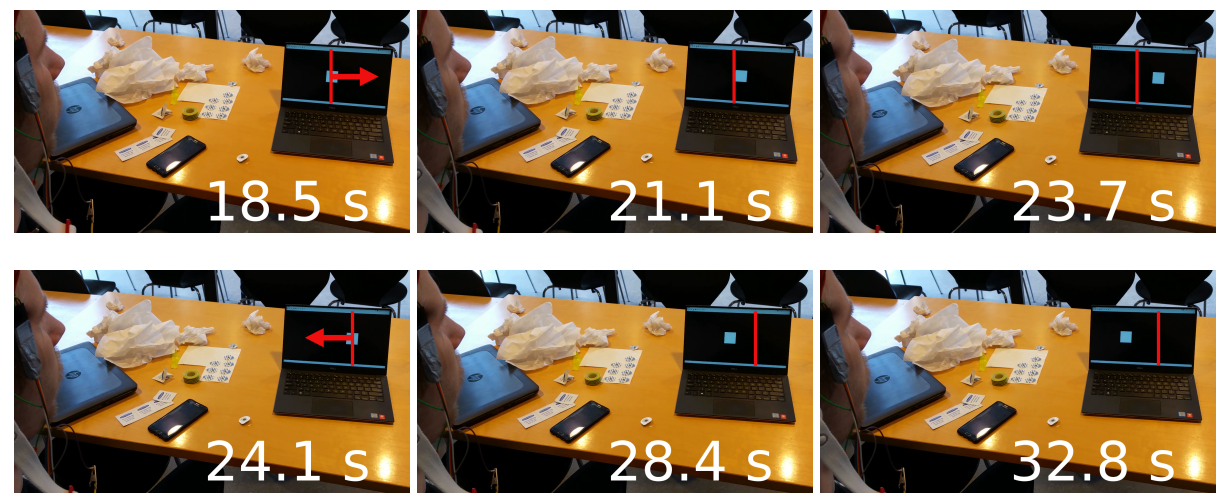

Fig. 7. Snapshots of the real-time experiment with their respective timings. Top row shows right hand MI and bottom row shows left hand MI. The vertical red line indicates the initial square position in each row. The video of the experiments can be seen at www.manoonpong.com/SMC2018/video.mp4

In the experiment, live visual feedback in the form of a white square target on a black background was available to the subject. A valid label produced by the classifier caused the target to move a short distance in the left or right direction, depending on the predicted class. If no valid label was produced, the target stayed still. The results of the real-time experiment are shown as snapshots in Fig. 7.

The implemented system was responsive and exhibited no noticeable lag between subject mental activity and target movement. The developed framework could process the provided input data stream in an efficient and timely manner, appropriate for the implemented real time application.

During testing, it was evident that the target movement was influenced by the subject's mental activity and was not merely a product of random noise. In the best cases, the subject could steadily move the target towards the left and right edges of the feedback screen through continuous focus on the appropriate MI class. However, the subject's ability to reliably control the target deteriorated significantly with increasing fatigue. As the subject lost focus, the target exhibited quivering behaviour due to random misclassification.

In the best cases, the subject was able to steadily control movement of the target through motor imagery, which proves that the system could in principle already be used in real life applications. It would, however, still need a considerable amount of tuning before any such usage as its performance seemed quite unstable in a number of trials.

The task at hand was physically and mentally tiring for the subject because a high level of focus and minimal body movement were crucial for a successful trial. As the subject grew increasingly fatigued with time, he lost control over the target. The cause of this might be in different brain activity patterns 
that appeared when the subject was tired and could not be interpreted by the system. The performance issue could therefore be partially resolved through subject training and collecting additional data to train the classifier, as well as to determine the subject's fatigue level and adjust the processing accordingly.

Future work will be focused on introducing adaptive methods with online adjustment of the control and feature classification parameters [19].

\section{Conclusion}

This paper presents the development of a real-time motor imagery based EEG brain-machine interface. Several filtering, feature extraction, and classification methods were investigated, tested and compared with three data sets obtained through different recording methods, with varying amounts of environmental noise. The experiments show that band power features based on IIR filters, paired with the one-versus-all LDA classifier give the best classification accuracies while maintaining a comparably high level of robustness.

The best performing signal processing and classification methods were tested in real-time, using wireless hardware with dry electrodes in a noisy environment. The results were promising, showing that the interface performs well even with an untrained subject.

\section{Acknowledgement}

This work is supported by Centre for BioRobotics (CBR) at University of Southern Denmark (SDU, Denmark) and Horizon 2020 Framework Programme (FETPROACT-01-2016-FET Proactive: emerging themes and communities) under grant agreement no. 732266 (Plan4Act).

\section{References}

1. Berger Neurorobotics. Available: http://bergerneurorobotics.com/, accessed: 10. 3.2018

2. Slovenian Initiative for National Grid (SLING). Available: http://www.sling.si, accessed: 10. 3. 2018

3. Alessio, S.M.: Digital Signal Processing and Spectral Analysis for Scientists. Springer International Publishing (2016)

4. Amzica, F., Lopes da Silva, F.H.: Cellular substrates of brain rhythms, pp. 33-63. Wolters Kluwer - Lippincott Williams \& Wilkins, 6th edn. (2011)

5. Blankertz, B., Dornhege, G., Krauledat, M., Schroeder, M., Williamson, J., Murray-Smith, R., Müller, K.R.: The Berlin Brain-Computer Interface presents the novel mental typewriter Hex-o-Spell. 3rd International BCI Workshop and Training Course, Graz, 2006, Graz, Austria(2006). pp. 2-3 (2006), http://eprints.pascal-network.org/archive/00002463/\{\\%\}5Cnhttp: //eprints.nuim.ie/1786/

6. Brunner, C., Leeb, R., Müller-Putz, G., Schlögl, A., Pfurtscheller, G.: BCI Competition 2008 - Graz data set A (2008) 
7. Goodfellow, I., Bengio, Y., Courville, A.: Deep Learning. MIT Press (2016), http: //www. deeplearningbook.org

8. Hastie, T., Tibshirani, R., Friedman, J.: The Elements of Statistical Learning. Springer Series in Statistics, Springer New York Inc., 2nd edn. (2009)

9. Hjorth, B.: EEG analysis based on time domain properties. Electroencephalography and Clinical Neurophysiology 29(3), 306-310 (1970)

10. Kingma, D.P., Ba, J.: Adam: A method for stochastic optimization. CoRR abs/1412.6980 (2014), http://arxiv.org/abs/1412.6980

11. Klem, G.H., Lüders, H.O., Jasper, H.H., Elger, C.: The ten-twenty electrode system of the International Federation 44195, 2-5 (1999)

12. McFarland, D., Wolpaw, J.: Eeg-based brain-computer interfaces. Current Opinion in Biomedical Engineering 4, $194-200$ (2017), http://www.sciencedirect. com/science/article/pii/S246845111730082X, synthetic Biology and Biomedical Engineering / Neural Engineering

13. Naeem, M., Brunner, C., Leeb, R., Graimann, B., Pfurtscheller, G.: Seperability of four-class motor imagery data using independent components analysis. Journal of Neural Engineering (3), 208-216 (2006)

14. Pfurtscheller, G., Lopes Da Silva, F.H.: Event-related EEG/MEG synchronization and desynchronization: Basic principles. Clinical Neurophysiology 110(11), 18421857 (1999)

15. Pfurtscheller, G., Neuper, C., Guger, C., Harkam, W., Ramoser, H., Obermaier, B., Pregenzer, M.: Current Trends in Graz Brain-Computer Interface (BCI) Research. IEEE Transactions on Biomedical Engineering 8(2), 216-219 (2000)

16. Pfurtscheller, G., Brunner, C., Leeb, R., Scherer, R., R., M.P.G., Neuper, C.: The Graz Brain-Computer Interface, pp. 79-96. Springer (2010), http://link. springer.com/10.1007/978-3-642-02091-9

17. Pfurtscheller, G., Neuper, C.: Dynamics of Sensorimotor Oscillations in a Motor Task, pp. 47-64. Springer (2010), http://link.springer.com/10.1007/ 978-3-642-02091-9

18. Prakaksita, N., Kuo, C.Y., Kuo, C.H.: Development of a motor imagery based brain-computer interface for humanoid robot control applications. In: 2016 IEEE International Conference on Industrial Technology (ICIT). pp. 1607-1613 (March 2016)

19. Sun, S., Zhou, J.: A review of adaptive feature extraction and classification methods for EEG-based brain-computer interfaces. Proceedings of the International Joint Conference on Neural Networks pp. 1746-1753 (2014)

20. Tibor Schirrmeister, R., Springenberg, J.T., Fiederer, L.D.J., Glasstetter, M., Eggensperger, K., Tangermann, M., Hutter, F., Burgard, W., Ball, T.: Deep learning with convolutional neural networks for brain mapping and decoding of movement-related information from the human EEG. ArXiv e-prints (Mar 2017), https://arxiv.org/abs/1703.05051v1

21. Widmann, A., Schröger, E., Maess, B.: Digital filter design for electrophysiological data - a practical approach. Journal of Neuroscience Methods (2014)

22. Wolpaw, J.R., McFarland, D.J., Vaughan, T.M.: Brain-computer interface research at the Wadsworth Center. IEEE Transactions on Rehabilitation Engineering 8(2), 222-226 (2000)

23. Wolpaw, J.R., Birbaumer, N., McFarland, D.J., Pfurtscheller, G., Vaughan, T.M.: Brain-computer interfaces for communication and control. Clinical neurophysiology : official journal of the International Federation of Clinical Neurophysiology 113(6), 767-91 (2002), http://www.ncbi.nlm.nih.gov/pubmed/12048038 